\title{
Discurso Matemático Escolar y Exclusión. Una Visión Socioepistemológica
}

\section{Academic Mathematical Discourse and Exclusion. \\ A Socio-epistemological Vision}

\author{
Daniela Soto* \\ Ricardo Cantoral $^{* *}$
}

\begin{abstract}
Resumen
El artículo identifica, caracteriza y ejemplifica al fenómeno de la exclusión provocado por el discurso Matemático Escolar (dME), de ahí que aborde como tema central la discusión que se puede desarrollar en torno a quién y cómo se excluye en el acto educativo. Consideramos a la exclusión en un sentido más amplio que el proceso de afectación del individuo al quedar fuera de determinado grupo o carecer de cierto derecho, sino al hecho de tener acceso sin ser considerado, a ser invisible. El $d M E$ se caracteriza por lo hegemónico y utilitario, desprovisto de marcos de referencia, con lo cual impone significados, argumentos y procedimientos centrados en los objetos matemáticos. Con ello elude el carácter funcional del saber matemático, dado que es el individuo quien, en su comunidad, lo construye. Por tanto, sostenemos que el discurso nos ha excluido de construir conocimiento matemático.
\end{abstract}

Palabras-clave: Exclusión. Discurso Matemático Escolar. Socioepistemología. Violencia Simbólica. Sistema de Razón.

\begin{abstract}
The paper identifies, characterizes, and illustrates the phenomenon of exclusion caused by the academic mathematical discourse $(d M E)$, hence addressing the main subject of discussion that may develop on who and what is excluded in the educational act. We consider exclusion in a broader sense that the process of involvement of the individual to be out of certain group or lack certain rights, but because of access without being considered to be invisible.

The $d M E$ is characterized by the hegemonic and utilitarian, devoid of reference frames, which imposes meanings, arguments, and procedures focused on mathematical objects. This avoids the functional nature of mathematical knowledge since the individual person builds it in their community. Therefore, we hold that the discourse has excluded us to build mathematical knowledge.
\end{abstract}

Keywords: Exclusion. Scholar Mathematical Discourse. Socio-epistemology. Violence Symbolic. Reason System.

\footnotetext{
* Doctora en Ciencias en la Especialidad de Matemática Educativa por el Centro de Investigación y de Estudios Avanzados del Instituto Politécnico Nacional (CINVESTAV-IPN), Distrito Federal, México. Académica del Departamento de Matemática y Ciencia de la Computación de la Universidad de Santiago de Chile. Dirección postal: Las Sophoras n 173. Estación Central. Santiago de Chile.E-mail: daniela.soto.s@usach.cl.

"* Doctor en Ciencias en la Especialidad de Matemática Educativa por el Centro de Investigación y de Estudios Avanzados del Instituto Politécnico Nacional (CINVESTAV-IPN), Distrito Federal, México. Investigador Titular y Jefe del Departamento de Matemática Educativa del Centro de Investigación y de Estudios Avanzados del Instituto Politécnico Nacional (CINVESTAV), México, Distrito Federal. Dirección Postal: Av. Instituto Politécnico Nacional n. 2508, Col. San Pedro Zacatenco, 07360, México, DF. E-mail: rcantor@cinvestav.mx.
} 


\section{La problemática}

Este artículo reporta una investigación que identifica, caracteriza y ejemplifica el fenómeno de exclusión en la construcción del conocimiento matemático desde una perspectiva que juzgamos innovadora. Mostraremos que el discurso Matemático Escolar $(d M E)$ es un sistema de razón que produce una violencia simbólica.

Empezamos señalando que la región latinoamericana comparte una enorme tradición cultural a la par que sistemas educativos, con características y carencias similares. La problemática de la enseñanza y el aprendizaje de las matemáticas es una de ellas, la comunidad educativa encara estos problemas desde la perspectiva del fracaso. El llamado fracaso escolar se ha relacionado, principalmente, con el incumplimiento de objetivos de aprendizaje en matemáticas definidos por las instituciones educativas. Cesar de Mattos (2011, p.306) señala que "En la década de 1980 y 1990, cierto tipo de resistencia hacia el aprendizaje de la matemática escolar, reconocida en los estudiantes que no aprenden matemáticas, caracterizó el fenómeno del fracaso de la educación escolar en diversos países".

Si bien la perspectiva del fracaso escolar en el campo de las matemáticas escolares intenta no culpabilizar al estudiante, se tiende a encontrar las causas en las características del educando o bien del docente, ya sea al nivel de asuntos motivacionales, emocionales o cognitivos del estudiante o en las capacidades, conocimientos o creencias de las y los profesores entre otras (GÓMEZ, 1997; GUERRERO; BLANCO; GIRL, 2006; PALACIOS; HIDALGO; MAROTO, 1999). Así, hemos notado que el grueso de los análisis en la literatura, suelen personificar el fracaso en matemáticas. El profesor o el estudiante aparecen como los responsables únicos del desinterés o de los bajos rendimientos en la materia (ESCUDERO, 2005; PERRENOUD, 1990). Sin embargo, consideramos que otra perspectiva es posible, desde nuestro enfoque teórico nos hemos preguntado sobre las características del rol que juega la matemática escolar en dicho fracaso.

Por el lado de la disciplina de la Matemática Educativa ${ }^{1}$, se han constituido diversos marcos teóricos para interpretar estos fenómenos de enseñanza y de aprendizaje de las matemáticas. Algunos de los cuales responden a los asuntos propios de la dimensión cognitiva del aprendizaje en matemáticas: alusiva a las representaciones mentales o construcciones

\footnotetext{
${ }^{1}$ El nombre de Matemática Educativa da a nuestra disciplina una ubicación geográfica y conceptual: en el mundo anglosajón, el nombre que le han dado a las práctica social asociada es el de Mathematics Education, mientras que en la Europa continental le han llamado Didáctica de las Matemáticas, Didactique des Mathématiques, didaktik der Mathematik, por citar algunas de las escuelas más dinámicas (CANTORAL; FARFÁN, 2003).
} 
mentales de los conceptos, mientras que otros asumen la problemática en términos de las interacciones entre el saber, el alumno y el profesor, poniendo el énfasis en las situaciones específicas secuenciadas para el aprendizaje de tales conceptos matemáticos asociados (ROAFUENTES; OTACK, 2012; CONTRERAS; GARCIA; FONT, 2012; ALVARADO; GONZÁLEZ, 2013; ARAVENA; CAAMAÑO, 2013). Si bien todos estos enfoques teóricos son enormes contribuciones a la disciplina, su centración principal recae en los objetos matemáticos (conceptos y procedimientos matemáticos como categorías apriorísticas), es decir, los conceptos y las explicaciones se desarrollan en tales acercamientos, sin problematizar el saber en juego.

La teoría Socioepistemológica (CANTORAL, 1990), se diferencia de otros marcos teóricos en el campo de la Matemática Educativa, por problematizar al saber dentro de un paradigma epistemológico distinto, concebimos al conocimiento matemático como aquel que se genera a partir de las prácticas socialmente situadas. Lo que implica un estudio sistémico del discurso Matemático Escolar (dME) y genera propuestas para su rediseño, a partir de la construcción social del conocimiento matemático (CSCM) (SOTO, 2010; CORDERO; SILVA-CROCCI, 2012). De esta forma no atiende, exclusivamente, al aprendizaje de los conceptos y procesos matemáticos, sino que incorpora a la investigación dimensiones sociales, históricas, culturales e institucionales, que permiten efectivamente la construcción del conocimiento matemático. Así, la Socioepistemología incorpora la noción de práctica social para explicar el transito del conocimiento al saber.

Según Cantoral et al. (2006) se explica que el conocimiento matemático adquiere un estatus de saber sólo hasta que se haya socializado en ámbitos no escolares. Así también que, su difusión hacia y desde el sistema de enseñanza le obliga a una serie de transformaciones que afecta directamente a su estructura y su funcionamiento, de manera que afecta, también, las relaciones que se establecen entre los estudiantes y sus profesores. Es decir, el proceso de constitución del conocimiento tiene una componente social, que va más allá de la interacción humana para construir funciones mentales (en el sentido Vigotskiano), damos cuenta de cómo las formaciones sociales se organizan para dar una institucionalidad al saber, donde se ponen en juego intensos procesos de negociación y de debate.

En el intento por difundir esos saberes se conforman discursos, que la Socioepistemología ha denominado con el término $d M E$, aclaran que la estructura de dichos discursos no se reduce a la organización de los contenidos temáticos, ni a su función declarativa en el aula (el discurso escolar), sino que se extiende un tanto más allá, al llegar al 
establecimiento de bases de comunicación para la formación de consensos y la construcción de significados compartidos (CANTORAL et al., 2006).

Las investigaciones que se han ocupado del $d M E$ para nociones específicas (BUENDÍA, 2006, 2011; CANTORAL 1990, CANTORAL et al., 2006; CORDERO, 2003, 2008; FARFÁN, 2012; MONTIEL, 2011; entre otras), han evidenciado una serie de características que enunciamos a continuación y que fueron analizadas en profundidad en Soto (2010):

- La atomización en los conceptos: no se consideran los contextos sociales y culturales que permiten la constitución del conocimiento.

- El carácter hegemónico: existe una supremacía de argumentaciones, significados y procedimientos, frente a otras.

- La concepción de que la Matemática es un conocimiento acabado y continúo: los objetos matemáticos son presentados como si hubiesen existido siempre y con un orden.

- El carácter utilitario y no funcional del conocimiento: la organización de la matemática escolar ha antepuesto la utilidad del conocimiento a cualquiera de sus restantes cualidades. Se busca que el conocimiento tenga un carácter funcional, en el sentido que logre integrar tal conocimiento a la vida para transformarla.

- La falta de marcos de referencia para resignificar la matemática escolar: se ha soslayado el hecho de que la Matemática responde a otras disciplinas y, por tanto, es ahí donde encuentra una base de significados naturales.

Por separada, cada una de estas características permite tratar al $d M E$, mientras que, en su conjunto, brindan una información novedosa, pues permite reconocer y estudiar al fenómeno de exclusión. Con estas características, reconocemos al $d M E$ como impositivo, donde el conocimiento matemático aparece en forma estática, no susceptible de construcción o modificación de parte del individuo. Es decir, profesores y estudiantes aparecen como comunicadores y aprendices de un conocimiento legítimo socialmente, sin contar con la posibilidad de construirlo o modificarlo. Esta consideración nos lleva a ampliar la visión del fracaso hacia otra, típicamente social, como lo es el fenómeno de la exclusión.

La forma de exclusión que reportamos en este artículo se refiere a la imposición de argumentaciones, significados y procedimientos asociados a los objetos matemáticos que ha promovido el $d M E$ y que induce el que los actores didácticos sean excluidos de la construcción del conocimiento matemático. 


\section{Marco teórico}

Problematizar cómo y por qué la matemática escolar genera un tipo de exclusión, parte de la reflexión que pone en la palestra la Teoría Socioepistemológica. La concepción de la Matemática que subyace en la enseñanza tradicional ha estado dada históricamente en términos de lo apriorístico, lo preexistente, es decir, se ha considerado independiente de las prácticas que le acompañan, el conocimiento matemático se ha concebido como un sistema de verdades seguras, no modificable por la experiencia humana (ESPINOZA, 2009). Esto ha generado que en la planeación y ejecución de la enseñanza el individuo sea marginado parcial o totalmente del proceso de su construcción.

La Socioepistemología propone dar un vuelco a la visión tradicionalista de la matemática escolar. En términos concretos, propone el cambio - a nivel institucional y cotidiano - de la visión que ubica a los objetos matemáticos, metafóricamente, en un altar y que conduce nuestra atención hacia cómo estos son aprendidos por el estudiante, para convocarnos al estudio de la actividad humana y las prácticas sociales, pues estas están en la base de la CSCM.

Las prácticas sociales tienen, como característica fundamental, establecer las normas del funcionamiento social (función normativa). Ello exige que en forma articulada sean simultáneamente funcionales para los individuos en comunidad (función pragmática), permitan la comunicación entre los miembros (función discursiva) y constituyan identidades (función identitária); por tanto, el estudio de la CSCM se da en los usos del conocimiento matemático por construir situaciones de aprendizaje centradas en elementos provenientes de las prácticas de la comunidad estudiada, y no sólo de las características de los propios objetos matemáticos. Por tanto, de manera natural, creemos que el rediseño del $d M E$ a partir del enfoque socioepistemológico incluirá al individuo situado en el proceso de construcción.

Cuando se teoriza particularmente sobre la exclusión, se suele atribuir el término a un proceso o condición - dependiendo de la perspectiva - ligado con diferentes grupos o individuos que se encuentran al margen de ciertos derechos sociales, civiles, educacionales, entre otros.

Dentro de la Matemática Educativa el fenómeno de la exclusión ha sido poco estudiado y documentado, sin embargo podemos reconocer dos focos de interpretación: el 
primero se preocupa de cómo las prácticas pedagógicas y el currículo de matemáticas no representan algunos grupos sociales, motivo por el cual se produce una exclusión hacia esos sectores (KNIJNIK, 2007; RIVAS, 2005; FERNANDES; HEALY, 2010). El segundo se centra en que la sociedad excluye con la Matemática, porque sin saber matemáticas, no podemos formar parte del breve porcentaje de personas privilegiadas (GIMÉNEZ; DIEZPALOMAR; CIVIL, 2007).

Las investigaciones sobre exclusión en la educación, en general, se destacan dos planteamientos distintos que nuestra investigación entrelaza. El primero tiene relación con una forma de excluir a los estudiantes a partir de la imposición de significados, socialmente establecidos y legitimados; Bourdieu y Passeron (2005) denominaron a este tipo de exclusión con el término violencia simbólica. El segundo se refiere a la discusión que se puede llegar a desarrollar en torno a quién excluye. Existe una perspectiva que plantea que la exclusión es producto de los sistemas de razón (conocimiento) que subyacen a las prácticas y representaciones sociales de los individuos. Estos sistemas de razón se pueden ver como mapas que delinean lo normal, norman, y por tanto, excluyen por omisión todo aquello que quede fuera. A continuación, caracterizaremos brevemente estas dos componentes, a saber: la violencia simbólica y los sistemas de razón, con el fin de presentar este modelo de exclusión.

\subsection{La violencia Simbólica}

Para los sociólogos, según Littlewood (2005), existen dos nociones de exclusión en la escuela; la primera entiende la escolarización y la educación formal no sólo como algo que tiene que ver con la transmisión de conocimiento, sino también con la selección. Pero toda selección supone no sólo identificar ciertos candidatos como aceptables para su inclusión, sino también identificar a los que no son aceptables y rechazarlos o excluirlos. La segunda es un tipo más sutil de exclusión, y tiene que ver con los educadores que intentan convencer a los excluidos de que no se trata del sistema que los excluye, sino más bien de ciertas características o déficits que tienen los propios candidatos.

El segundo tipo de exclusión queda ilustrada en la obra de Bourdieu y Passeron (2005). Los autores introducen el término violencia simbólica para describir "todo poder que logra imponer significaciones e imponerlas como legítimas disimulando las relaciones de fuerza en que se funda su propia fuerza" (BOURDIEU; PASSERON, 2005, p.44). "La violencia simbólica es esa violencia que arranca sumisiones que ni siquiera se perciben como 
tales apoyándose en unas "expectativas colectivas", en unas creencias socialmente inculcadas" (BOURDIEU, 1997, p.173).

Bourdieu (1997) conceptualiza los diferentes sistemas simbólicos como el arte, la religión, la ciencia y el mismo lenguaje a partir de la noción de arbitrario cultural. Fernández (2005) señala que para Bourdieu los sistemas simbólicos realizan, simultáneamente, tres funciones interrelacionadas, pero diferentes: conocimiento, comunicación y diferenciación social.

El poder de estos sistemas simbólicos no emplea la violencia física sino la violencia simbólica. Es un poder legitimador que suscita el consenso tanto de los dominadores como de los dominados, un "poder que construye un mundo" en cuanto supone la capacidad de imponer la "visión legítima del mundo social y de sus divisiones" (BOURDIEU, 1987, p.13) y la capacidad de imponer los medios para comprender y adaptarse al mundo social mediante un sentido común, contribuyendo, así, a la reproducción intergeneracional de acuerdos sociales. Fernández (2005, p.9) señala que:

El poder simbólico es un poder «invisible», que no es reconocido como tal, sino como algo legítimo, presupone cierta complicidad activa por parte de quienes están sometidos a él, requiere como condición de su éxito que éstos crean en su legitimidad y en la de quienes lo ejercen.

Para Bourdieu y Passeron (2005, p.45) la acción pedagógica (AP) apoya a la violencia simbólica relativa a la educación. Ellos señalan que "la AP es objetivamente una violencia simbólica en tanto que impone, por un poder arbitrario, una arbitrariedad cultural". En términos concretos la AP se puede resumir como las diferentes formas de transmitir el conocimiento a través de una diversidad de conductos. Conocimiento que, a su vez, se entiende como una arbitrariedad cultural. En este sentido señalan:

La selección de significados que define objetivamente la cultura de un grupo o de
una clase como sistema simbólico es arbitraria en tanto que la estructura y las
funciones de esa cultura no se pueden deducir de ningún principio universal, físico,
biológico o espiritual, puesto que no están unidas por ningún tipo de relaciones
internas a la "naturaleza de las cosas" o a una "naturaleza humana" (BORDIEU;
PASSERON, 2005, p.48).

Por otro lado, la AP no podría cumplir con sus objetivos sin una autoridad pedagógica (AuP). Es decir, que las formas de transmisión del saber son válidas en tanto que exista una autoridad que legítima desde qué, cómo y cuándo se debe de enseñar tal o cual conocimiento. "La AP implica necesariamente como condición social para su ejercicio la AuP y la autonomía relativa de la instancia encargada de ejercerla" (BOURDIEU; PASSERON, 2005, p. 52). En otras palabras, toda AP necesita legitimar la inculcación de cierto conocimiento y un mecanismo necesario es la legitimidad del emisor (libros, profesor, currículo). 
En conclusión, la violencia simbólica es una forma de excluir a través de la imposición. En este proceso los agentes educativos mantienen el orden, es decir, contribuyen a la reproducción de las relaciones de poder prevalecientes en la sociedad contemporánea por medio de la enseñanza y de la selección de los alumnos, al convencerlos de que interioricen la superioridad o justicia de la cultura o conocimiento dominante y la inferioridad de la cultura o conocimiento de los grupos y categorías sociales dominadas.

\subsection{Los sistemas de razón}

Popkewitz y Lindblad (2005, p.118) proponen dos enfoques teóricos para analizar el fenómeno de exclusión: La problemática de la equidad y la problemática del conocimiento.

Denominamos "problemáticas" a los diferentes enfoques, con el propósito de resaltar cómo los métodos de la ciencia y los conceptos y teorías de los asuntos sociales producen formas de pensar y ordenar la acción de comprender los resultados.

Para los estudios que atienden la problemática de la equidad el objetivo fundamental está relacionado con la representación, es decir, en cómo se comprende y explica la participación/no participación de los grupos, poblaciones o individuos, clasificados típicamente mediante categorías de raza, género o clase social, si están representados o no.

En contraste con la problemática de la equidad, que examina cuestiones de representación y acceso de individuos y grupos a las prácticas educativas, la problemática del conocimiento se centra en los sistemas de razón que organizan los enfoques de resolución de problemas en las prácticas educativas. Popkewitz y Lindblad (2005) señalan que esta problemática enfoca al conocimiento no como algo que representa un interés de grupo, sino como sistemas construidos socialmente que clasifican, diferencian y dividen los objetos del mundo y lo que debe ser conocido.

El conocimiento, pues, no es un mecanismo descriptivo producido mediante propiedades estructurales para incluir o excluir grupos de personas, como una clase. El conocimiento es más bien una práctica social que estructura el campo de acción posible, al prever la conductas mediante la inscripción de principios de rendimiento y de modo de subjetivación, es decir, de las reglas de acuerdo con las cuales se comportan los individuos como responsables, automotivados y competentes (DEAN, 1995, citado en Luengo, 2005, p.145).

La problemática del conocimiento se centra, principalmente, en el conocimiento como una práctica material. Pero este interés por lo material del conocimiento no se refiere a centrarse en las representaciones de los grupos de excluidos, sino en cómo los sistemas de razón han generado históricamente principios de diferenciación y división, a través de los 
cuales se construyen las representaciones de los actores y sus normas de acción. En otras palabras, las investigaciones que atienden la problemática del conocimiento no se preocupan de la raza, el género o la clase social, sino en entender cómo el conocimiento produce ese tipo de categorías.

Un sistema de razón se puede explorar mediante la analogía de un mapa de carretera. Del mismo modo que un mapa de carretera nos informa las distancia y los caminos para viajar, y lo que queda dentro y fuera de los territorios, un sistema de razón nos habla simbólicamente de cómo ordenar y dividir los objetos sobre los que se debe reflexionar, nos dice lo que hay dentro de la razón, y al mismo tiempo inscribe lo que está afuera de la misma, con lo cual excluye por medio de los instrumentos de ordenación y diferenciación de la razón aplicada a la solución de las problemáticas. Es decir, un sistema de razón se caracteriza por crear mapas en los cuales delinea las formas de actuar, razonar, dar significados y/o argumentar de los individuos, entre otras. Por tanto, un modo alternativo quedará fuera de los límites, considerándose extraño, anormal o inadecuado.

\section{El modelo de exclusión}

Primeramente, debemos aclarar que nuestro estudio plantea que la exclusión producida por la Matemática Escolar emerge de ella misma, es decir, consideramos que existe un sistema de razón que fundamenta a la organización de la Matemática Escolar y que genera principios donde el quehacer de los individuos o de los grupos queda al margen de la construcción del conocimiento. Por este motivo nuestra investigación se ubica dentro de los estudios que atienden la problemática del conocimiento, según fueron clasificados por Popkewitz y Lindblad (2005). Por tanto, en el sentido metodológico nos planteamos hacer análisis de ese sistema de razón al que denominamos el $d M E$.

Ahora bien, ¿qué tipo de exclusión pretendemos evidenciar? Nuestro estudio pretende mostrar cómo el $d M E$ genera violencia simbólica. Es decir, planteamos que es el propio conocimiento con fines didácticos el que impone significados y valida sólo un tipo de argumentación, lo que genera un tipo muy sutil de exclusión, donde los actores del sistema educativo (estudiantes, profesores, padres, directivos, políticos etc.) son cómplices involuntarios debido a la legitimidad de la cual goza el sistema que lo produce.

De esta forma, nuestra investigación combina los dos tipos de análisis de la exclusión escolar que hemos descritos. Por una parte, explicitaremos las características del sistema de 
razón-dME- que fundamenta a la organización de la Matemática Escolar, el cual delimita lo que queda dentro de lo normal en la enseñanza y el aprendizaje de la matemática. Y por otra, evidenciaremos cómo ese sistema de razón a partir de sus características y de la legitimidad social de la cual goza, impone significados, procedimientos y argumentaciones que los actores del sistema didáctico reconocen e interiorizan, reconociendo en ellas hegemonía y superioridad. Veamos, a continuación, el modelo de exclusión propuesto que articula nociones usualmente disjuntas: discurso Matemático Escolar $(d M E)$, Sistema de razón $(S . R)$ y Violencia Simbólica (V.S), mediante la idea de mapa que explica la exclusión a través de la imposición:

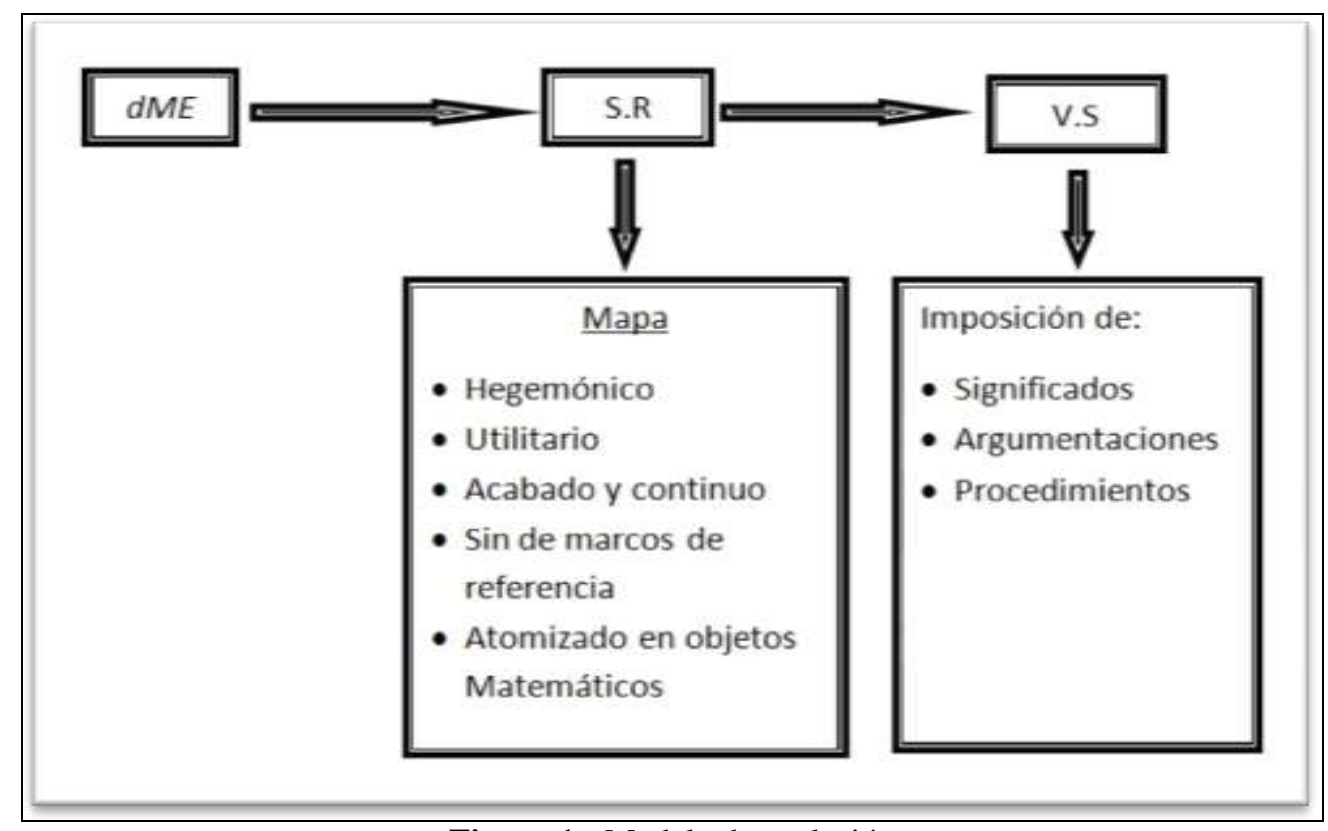

Figura 1 - Modelo de exclusión

El $d M E$ es un sistema de razón que produce violencia simbólica, a partir de la imposición de argumentaciones, significados y procedimientos. El S.R queda expresado en el mapa compuesto por las características del $d M E$.

A fin de ilustrar el uso del modelo teórico propuesto, mostraremos, a continuación, el análisis de una pieza de saber específico, que en el ámbito educativo se conoce como la enseñanza de algoritmos para ideas matemáticas complejas como lo es el tratamiento analítico de las formas indeterminadas. Específicamente, nos referimos al tratamiento escolar de la Regla de L'Hôpital de los cursos de cálculo diferencial.

\section{Metodología}


De acuerdo a nuestro marco teórico y al modelo de exclusión que hemos propuesto, probaremos la siguiente hipótesis: el $d M E$ es un sistema de razón que produce violencia simbólica. Para ello hemos desarrollado el análisis de un objeto matemático particular de la Matemática Escolar de nivel superior: el Teorema de L'Hôpital, con esto mostraremos la exclusión producida por el $d M E$ y cómo sus características conforman un mapa (figura 1) del S.R.

Para desarrollar el análisis de la exclusión ocasionada por el $d M E$ hemos decidido estudiarlo en los textos de estudio, ya que en ellos se materializa la visión que fundamenta a la Matemática Escolar. En Soto (2010) se considera al $d M E$ de los textos de estudio como las argumentaciones, significados y procedimientos invariantes, es decir que se repiten en las diferentes presentaciones. De esta forma, hemos revisado textos como Purcell y Varberg (1995), Larson, Hostetler y Edwards (1995) y Stewart (1999), entre otros. Sin embargo, para efectos de este escrito presentamos sólo un caso que representa lo invariantes del teorema de L'Hôpital en los textos de estudio.

También, en el grupo de investigación se ha desarrollado el análisis de la obra de L’Hôpital (1696) desde hace algún tiempo, como un ejemplo de objeto didáctico singular, con el fin de mostrar que existen, en otros escenarios, argumentaciones, significados y procedimientos distintos de lo que presenta el $d M E$ (CAMBRAY, 1993; CASTAÑEDA, 2006; FLORES, 1989; SOTO; REYES, 2011).

\section{Análisis de los datos}

La exposición del teorema de L'Hôpital en el $d M E$ obedece a un tipo de argumentación: el cálculo del límite cuando la función es indeterminada en un punto. Este es el hilo conductor que norma las formas por las cuales el conocimiento se presenta y responderá a los porqués de su utilidad. En este sentido, la regla aparece como un objeto matemático útil para la resolución de ejercicios, relativos al límite.

Un ejemplo es la presentación del teorema en el texto de Stewart (1999). En él, el teorema de L'Hôpital es presentado como una regla, de la siguiente forma:

Regla de de L’Hôpital: Supóngase que $f$ y $g$ son derivables y que g’(x)¥ 0 cerca de $a$ (excepto quizás en $a$ ). Supóngase que

$$
\lim _{x \rightarrow a} f(x)=0 \quad y \quad \lim _{x \rightarrow a} g(x)=0
$$

O que $\quad \lim _{x \rightarrow a} f(x)= \pm \infty y \quad \lim _{x \rightarrow a} g(x)= \pm \infty$

(En otras palabras, tenemos una forma indeterminada del tipo $\frac{0}{0}$ o del $\underset{m}{m}$ )

$$
\lim _{x \rightarrow a} \frac{f(x)}{g(x)}=\lim _{x \rightarrow a} \frac{f^{\prime}(x)}{g^{\prime}(x)}
$$


Figura 2 - Presentación del Teorema de L’Hôpital en Stewart (1999)

El $d M E$ del teorema, como se refleja en el enunciado anterior, encuentra sus significados en el límite y el cociente de funciones. En este sentido, señalamos que el $d M E$ se encuentra centrado en los objetos matemáticos. Estos significados provienen de objetos matemáticos enseñados y supuestamente aprendidos con anterioridad, no se considerar argumentaciones, significaciones y procedimientos desde otros contextos de significación, sino que se imponen en el sentido de Bourdieu y Passeron (2005). El procedimiento que se asocia al teorema se encuentra en los procesos matemáticos de derivación.

Ahora bien, nuestra investigación pretende hacer ver (ejemplificando con este caso) que las argumentaciones, los significados y los procedimientos del cual nos provee el $d M E$ son impuestos. Por lo cual, no permiten al actor del sistema didáctico incluirse en la construcción del conocimiento matemático. En este sentido, es válido preguntarse ¿existirán otros marcos de referencia para resignificar el conocimiento?

Un marco de referencia puede ser la naturaleza del saber, es por esta razón que hemos estudiado la obra del Marqués de L’Hôpital. En este análisis queremos resaltar la situación que hizo emerger el teorema, la cual es soslayada en el $d M E$, y que provee de una argumentación, significados y procedimientos, no tan sólo diferentes, sino que podrían darnos elementos suficientes para rediseñar el $d M E$ del teorema en cuestión.

La obra de L'Hôpital está fuertemente influenciada por el cálculo leibniziano, los infinitesimales y la noción de diferencial así como del análisis cartesiano, es decir, del estudio de las curvas mediante métodos algebraicos.

En la sección IX, § 163 de la obra del Marqués de L’Hôpital (1696,). Se enuncia el siguiente problema.

Sea $\mathrm{AMD}$ una línea curva $(\mathrm{AP}=x, \mathrm{PM}=y, \mathrm{AB}=a)$ tal que el valor de la ordenada $y$ esté expresado por una fracción, en el cual el numerador y el denominador se vuelvan cada uno cero cuando $x=a$, es decir, cuando el punto $\mathrm{P}$ caiga sobre el punto dado B [fig.130]. Se pregunta cuál debe ser entonces el valor de la ordenada BD.

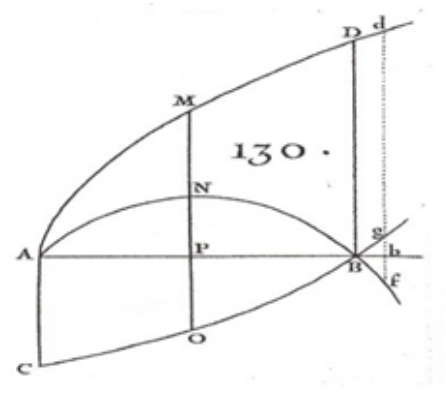


Figura 3 - Traducción del problema (L’Hôpital, 1998, p.259)

El problema que presenta L’Hôpital nos plantea una situación gráfica, donde dos curvas $(f(x)$ y $g(x))$, cuyo cociente que conforma a otra curva $(h(x)=f(x) / g(x))$, se interceptan en el eje de las $X$. preguntándose qué sucede con esa curva $h(x)$ en ese punto (Figura 4).

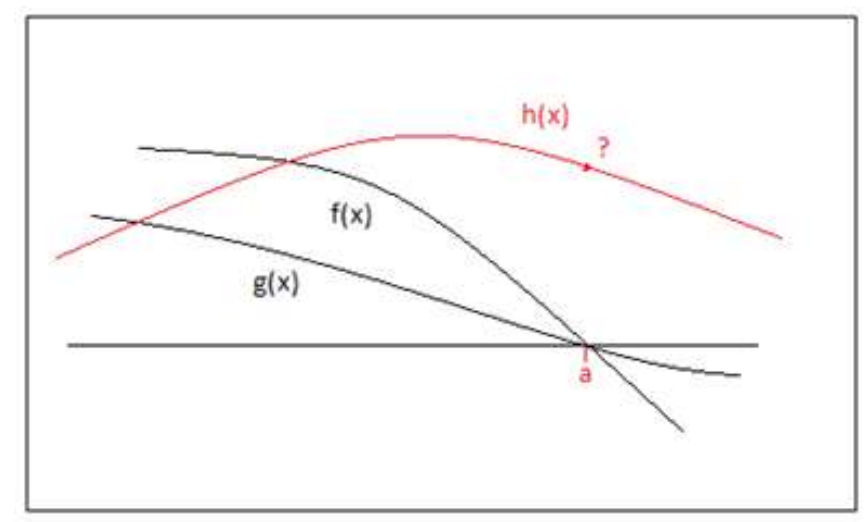

Figura 4 - Representación de la problemática de L’Hôpital

Debemos considerar que éste no sería un problema si las curvas, cuyo cociente representa a la tercera curva, no se interceptaran en el eje de las $X$. Por tanto, la argumentación que genera la situación es, justamente, la intercepción de dos curvas en el eje de las $X$ cuyo cociente será representada en una tercera curva.

Este es el hilo conductor de la situación y la problemática es encontrar el valor en ese punto de intercepción de la tercera curva. Ahora bien, si consideramos la solución que plantea L'Hôpital (Figura 5), podremos notar que tanto los significados como los procedimientos emergen de la situación gráfica.

Siendo ANB y $C O B$ dos líneas curvas conocidas que tienen a la línea $A B$ como eje común, tal que la aplicada PN, y tales que la ordenada PN exprese el numerador y la ordenada PO el denominador de la fracción general que conviene a todas las $P M$, de modo que $P M=\left(A B^{*} P N\right) / P O$. Es claro que estas dos curvas se intersectarán en el punto $B$, dado que, por la suposición, PN y PO se vuelven cada una cero cuando el punto $P$ cae en B. Planteado eso, si se concibe una ordenada bd infinitamente próxima a $B D$, y que intersecta a las líneas curvas $A N B$ y $C O B$ en los puntos $f$ y $g$, se tendrá $b d=(A B * b f) / b g$. la cual no difiere de $B D$. Entonces el problema consiste en encontrar la razón entre bg y bf. Ahora bien, es claro que al volverse $A B$ la abscisa $A P$, las ordenadas $P N$ y $P O$ se vuelven nulas, y que al volverse $A b$ la abscisa $A P$, se vuelven bf y $b g$. De donde sigue que estas ordenadas, las mismas bf y bg hacen la diferencia de las ordenadas en $B$ y $b$ con relación a las curvas ANB y COB, y por lo tanto, si se toma la diferencia de numeradores y se divide entre la diferencia del denominador, después de haber hecho $x=a=A b=A B$, se tendrá el valor buscado de la ordenada bd o $B D$. Lo cual se quería encontrar.

Figura 5 - Traducción de la solución del problema en la obra de L'Hôpital (L'Hôpital, 1998, p.259-260)

Los significados asociados son: la intersección de dos curvas, el cociente y la predicción. Cada uno de ellos aportará los elementos necesarios para la elección del 
procedimiento a seguir, en este caso: el cálculo de diferenciales; desde donde emergerán objetos y procesos matemáticos, a saber: la regla de L'Hôpital.

Ahora bien, después de haber identificado el tipo de situación del cual emerge el teorema y evidenciado que la argumentación del $d M E$, no sólo, no es la única sino que, además, impone a través de su legitimidad los significados y procedimientos, nos planteamos el considerar el mapa del $d M E$ que genera una violencia simbólica, identificando sus componentes en este ejemplo concreto.

Comencemos con el carácter hegemónico. Este se refiere a cómo el $d M E$ privilegia un sólo tipo de argumentaciones, significados y procedimientos. Como hemos visto, en el análisis del $d M E$ del teorema de L'Hôpital, la argumentación que se impone es la utilización de una regla para resolver ejercicios del límite cuando éstos no se pueden calcular de manera tradicional. De esta situación se desprenden significados y procedimientos, también impuestos, ya que la presentación obedece a una regla que se debe aplicar.

En nuestro análisis de la obra de L'Hôpital hemos encontrado otro tipo de argumentación, de la cual nacen los significados y los procedimientos a utilizar, en este caso de la génesis del problema, lo histórico, pero también podríamos encontrar argumentaciones provenientes de otras prácticas de referencia y de lo que Gómez (2009) y Zaldívar (2009) llaman cotidiano, que podrían resignificar este conocimiento.

En definitiva, con el análisis del Teorema de L’Hôpital evidenciamos el carácter hegemónico del $d M E$. En el sentido de que existen otras formas de enfocar el conocimiento matemático y que nos llevan a una real construcción de él, sin embargo, no son habitualmente consideradas.

El carácter utilitario del conocimiento, se refiere a la visión de que la Matemática es un saber útil para encarar ciertas problemáticas. En este caso el $d M E$ presenta a dicho conocimiento como una regla exitosa para la resolución de ejercicios relacionados con el límite, en particular cuando la función es indeterminada. No permite al sujeto problematizar, inferir y trastocar el conocimiento. Sólo se prevé que el sujeto la aprenda para cuando se enfrente a problemas similares. Así, el conocimiento no toma un carácter funcional, en el sentido de que le permita al sujeto resignificarlo en otros contextos, ya que es una regla estática. Por ejemplo, problematizar curvas cuando se interceptan en puntos conflictivos de ser analizados, lo cual es la argumentación que hace emerger al teorema, permitiría proponer nuevas hipótesis, inferir, desarrollar diferentes procedimientos, y el teorema aparecería como 
un caso especial de ser estudiado, por tanto el $d M E$ no se centraría en el objeto matemático y se resignificaría.

La concepción imperante de que la Matemática es un conocimiento constituido y anterior a la práxis humana, se evidencia en la presentación del teorema como una regla. Esta presentación impone un objeto preexistente, lo que no permite preguntarse ¿por qué es posible que tal concepto se construya como se construyó? En este sentido, se obliga a asumir un objeto matemático.

La presentación de los conocimientos matemáticos como terminados y lineales hace ver un problema, su solución y su posible demostración lógica a través de la aplicación de tal objeto, no permite que nos propongamos modificar los objetos. Olvidando que el conocimiento está en constante cambio y resignificación. No permite que nos plantemos modificar los objetos. Es decir, no le promueve que los individuos planteen nuevas hipótesis, inferir y transformar las situaciones que hacen emerger el conocimiento, en busca de otros significados sobre la actividad matemática.

La falta de marcos de referencia que obliguen a resignificar la Matemática Escolar, es evidente en el $d M E$ del Teorema de L'Hôpital. Por un lado, las explicaciones son construidas desde la matemática misma, no se consideran otros campos del conocimiento a los cuales la matemática responde; y por el otro, si bien la matemática puede ser el marco de referencia por el cual se construya el conocimiento matemático, en el ejemplo del Teorema de L'Hôpital podemos ver que sólo existe un tipo de argumentación que lo fundamenta. Por ejemplo, investigaciones como Tuyub y Cantoral (2012) hacen explicito que en las comunidades profesionales las gráficas son funcionales para su quehacer. En estos contextos la presencia del concepto de función es implícita, se manifiesta en las magnitudes, en los procesos de cambio y en como ocurren tales procesos. Esto nos podría brindar un nuevo marco de referencia en el cual se podría resignificar el teorema.

La atomización de los objetos y procesos matemáticos se manifiesta en una matemática escolar carente de argumentaciones y significados que provengan de la actividad humana. Se desconoce las prácticas de referencia que hacen emerger el conocimiento y las dinámicas institucionales que lo ponen en el lugar que ocupan. Esto nos impide tener una visión amplia de la estructura del saber, es decir, ¿cuáles son las situaciones que hacen que se construya dicho saber? ¿Por qué ese conocimiento y no otro? ¿Cuáles son sus usos? En este sentido plateamos que la atomización de los objetos y procesos matemáticos genera una exclusión hacia los actores del sistema didáctico. 
Con este ejemplo, y a partir de nuestro mapa de exclusión del $d M E$, hemos podido dilucidar que el $d M E$ presente en los textos de estudio generan una violencia simbólica, en el sentido de que imponen una única argumentación y que además los significados y procedimientos que emergen de ella giran en torno a los objetos matemáticos, no considerando el papel de los actores del sistema didáctico en la construcción de estos. Es en esta dirección que nuestras reflexiones se amplían sobre la necesidad del rediseño del $d M E$ para la eventual inclusión de los actores del sistema didáctico en la construcción del conocimiento matemático.

\section{A manera de conclusiones}

En este trabajo hemos alcanzado nuestros objetivos: identificar, caracterizar y ejemplificar el fenómeno de la exclusión que provoca la propia matemática escolar, desde una perspectiva innovadora en el campo de la Matemática Educativa; como dijimos, consideramos al $d M E$ como generador de dicho fenómeno. Hemos construido un modelo de exclusión, que permite caracterizar al $d M E$ como un sistema de razón, que excluye a los actores del sistema educativo (estudiante y docente o a los autores de texto, entre otros) de la construcción del conocimiento matemático a través de una violencia simbólica. Con el cual podrá analizarse otros episodios de construcción social con resultados similares y, en esa medida, tendremos más evidencia del papel que esta forma particular de exclusión juega en los procesos educativos.

El considerar al $d M E$ como un sistema de razón, como señalamos en las secciones anteriores, que delimita el campo de acción posible así como las representaciones sociales de los distintos actores podemos analizar el rol de los actores del sistema didáctico (profesor, el estudiante, el autor de los textos escolares, entre otros) como víctimas de una violencia simbólica.

Nuestro análisis del teorema de L'Hôpital, permitió mostrar, a manera de ejemplo, la imposición de argumentaciones, significados y procedimientos. Hemos evidenciado, por un lado, que su presentación en los textos de estudio es reducido a una regla que habrá de ser memorizada y aplicada en casos específicos de formas indeterminadas; por el otro, que existen otras argumentaciones que inducen la emergencia de significados y procedimientos asociados al saber y que no son consideradas en el propio $d M E$.

El considerar estas otras argumentaciones, significados y procedimientos, no sólo se trata de cambiar la estructura de los enunciados, ni asumir otras representaciones, sino de dar 
un papel primordial a la construcción social del conocimiento matemático, entendiendo que el conocimiento es un saber en uso y, por tanto, necesita incluirse al individuo en su construcción.

El haber identificado que el $d M E$ es excluyente - en tanto que impone y legitima esa imposición - conlleva al surgimiento de un nuevo argumento que reafirma la necesidad del rediseño del $d M E$ planteado por la teoría Socioepistemológica. La tarea de rediseñar no se debe confundir con la de reorganizar o reestructurar el saber matemático escolar, sino cambiar la centración hacia las prácticas sociales que hacen emerger el conocimiento. Asimismo, haber identificado que el $d M E$ produce una violencia simbólica y haber construido el mapa, proporcionamos algunos elementos para analizar el rediseño del $d M E$ y así, vigilar e intervenir en los casos que es pertinente.

Nuestro trabajo es relevante en dos sentidos, primero consideramos que observar y caracterizar este fenómeno nos permite cuestionar la epistemología dominante que se inscribe en el $d M E$ y proponer considerar las prácticas sociales como la centración en la Matemática Escolar da luces para resolver problemáticas como el fracaso escolar. Y segundo, creemos que es un aporte para la perspectiva teórica, la Socioepistemología, ya que es un argumento para la necesidad del rediseño que esta teoría propone a partir de la Construcción Social del Conocimiento Matemático.

Las limitaciones de esta investigación radican en las componentes metodológicas, en el sentido que es necesario ampliar el estudio hacia los agentes del sistema educativo, a saber: el profesor, el estudiante, los padres, escritores de textos entre otros, para poder robustecer y reafirmar el modelo de exclusión.

Los resultados de la investigación que se presentan en este artículo son parte de un primer acercamiento a un fenómeno social tan complejo como la exclusión. Se deben desarrollar más estudios en los ambientes de aprendizaje, en comunidades específicas, con lo cual se identifiquen y caractericen elementos que ayuden a robustecer y modificar el modelo de exclusión imperante.

\section{Referencias}

ALVARADO, A.; GONZÁLEZ, M-T. Generación interactiva del conocimiento para iniciarse en el manejo de implicaciones lógicas. Revista latinoamericana de investigación en Matemática Educativa, Ciudad de México, v. 16, n.1, p. 37-63, mar. 2013.

ARAVENA, M.; CAAMAÑO, C. Niveles de razonamiento geométrico en estudiantes de establecimientos municipalizados de la región del Maule, Talca, Chile. Revista latinoamericana de investigación en Matemática Educativa, Ciudad de México, v. 16, n.2, p.139-178. jul. 2013. 
BOURDIEU, J. The force of law -toward a sociology of the juridical field. Hastings Law Journal, San Francisco, v. 38, n. 5, p. 805-853, jul. 1987.

BOURDIEU, J. Razones prácticas: sobre la teoría de la acción. Barcelona: Anagrama.1997.

BOURDIEU, J.; PASSERON, J.C. La reproducción: elementos para una teoría del sistema de enseñanza. Ed. Traducción de Melendres J., Subirat M. Ciudad de México: Fontamara. 2005.

BUENDÍA, G. La construcción social del conocimiento matemático escolar: un estudio socioepistemológico sobre la periodicidad de las funciones. Madrid: Ediciones Díaz de Santos. 2011.

BUENDIA, G. Una socioepistemología del aspecto periódico de las funciones. Revista Latinoamericana de Investigación en Matemática Educativa, Ciudad de México, v. 9, n. 2, p. 227- 251, jul. 2006.

CAMBRAY, R. Procesos inherentes en la construcción del concepto de derivada: una exploración didáctica con un grupo de profesores de cálculo en servicio docente. 1993. 281f.Tesis (Maestría en Ciencias) - Departamento de Matemática Educativa del Centro de Investigación y de Estudios Avanzados, Instituto Politécnico Nacional, Ciudad de México, 1993.

CANTORAL, R. Categorías relativas a la apropiación de una base de significaciones propia del pensamiento físico para conceptos y procesos matemáticos de la teoría elemental de las funciones analítica. 1990. 554 f. Tesis (Doctorado en Ciencias) - Departamento de Matemática Educativa del Centro de Investigación y de Estudios Avanzados, Instituto Politécnico Nacional, Ciudad de México, 1990.

CANTORAL, R. et al. Socioepistemología y Representación: algunos ejemplos. Revista Latinoamericana de Investigación en Matemática Educativa, Ciudad de México, Número especial, p. 83-102, dic. 2006.

CANTORAL, R., FARFÁN, R.M. Matemática Educativa: Una visión de su evolución. Revista Latinoamericana de Investigación en Matemática Educativa, Ciudad de México, v. 6, n. 1, p. 27-40, mar. 2003.

CASTAÑEDA, A. Formación de un discurso escolar: el caso del máximo de una función en la obra de L’Höpital y Maria G. Agnasi. Revista Latinoamericana de Investigación en Matemática Educativa, Ciudad de México, v. 9, n. 2, p. 253-265, jul. 2006.

CESAR DE MATTOS, A. Educação Matemática e Sociedade. Boletim de educação Matemática, Rio Claro, v. 25, n. 41, p. 299-318, dic. 2011.

CONTRERAS, A., GARCIA, M. Y FONT, V. Análisis de un proceso de estudio sobre la enseñanza del límite de una función. Boletim de Educação Matemática, Rio Claro, v. 26, n. 42B, p. 667-690, abr. 2012.

CORDERO, F. Matemáticas y el cotidiano. In: Diplomado desarrollo de estrategias de aprendizaje paraas matemáticas del bachillerato. La transversalidad curricular de las matemáticas, 2013. Disponible en $<$ http://www.proyectosmatedu.cinvestav.mx/diplomado/mi_cuenta/data/pdfcordero/vid5/MATEMATICAS \&COTIDIANO,\%20ENE.2013..pdf>. Acceso en: 23, feb. 2013.

CORDERO, F. El uso de las gráficas en el discurso del cálculo escolar. Una visión socioepistemológica. In CANTORAL, R.; COVIÁN, O.; FARFÁN, R.M.; LEZAMA, J. Y ROMO, A. (Orgs.), Investigaciones sobre enseñanza y aprendizaje de las matemáticas: un reporte iberoamericano México: Díaz de Santos-Comité Latinoamericano de Matemática Educativa. A. C, 2008. p. 265-286.

CORDERO, F. Reconstrucción de significados del Cálculo Integral: La noción de acumulación como una argumentación. Ciudad de México: Iberoamérica. 2003. 
CORDERO, F. Y SILVA-CROCCI, H. Matemática Educativa, Identidad y Latinoamérica. El quehacer y la usanza del conocimiento disciplinar. Revista latinoamericana de Matemática Educativa, Ciudad de México, v. 15, n. 3, p. 295-318. Nov. 2012.

DEAN, M. Governing the unemployed self in an active society. Economy and Society, London, 1995, v. 24, n. 4, p. 559-583. Apud LUENGO, J. (Coord.). Paradigma de gobernación y de exclusión social en la educación; Fundamentos para el análisis de la discriminación escolar contemporánea. Barcelona, España: Pomaire. p.145. 2005.

ESCUDERO, J. Fracaso escolar, exclusión escolar: ¿De qué se excluye y cómo? Profesorado, Granada, v. 9, n.1, p.1-24. 2005.

ESPINOZA, L. Una evolución de la analiticidad de las funciones en el siglo XIX: un estudio socioepistemológico. 2009. 173f. Tesis (Maestría en Ciencias) - Departamento de Matemática Educativa Centro de Investigación y de Estudios Avanzados, Instituto Politécnico Nacional, Ciudad de México, 2009.

FARFÁN, R.M. Socioepistemología y Ciencia. Barcelona: Gedisa. 2012

FERNÁNDEZ, J.M. La noción de violencia simbólica en la obra de Pierre Bourdieu: una aproximación crítica. Cuadernos de trabajo social, Madrid, v. 18, p. 7-31. 2005.

FLORES, J. L'Hopital y lo rescatable de su obra para la enseñanza del cálculo. 1989. 174 f. Tesis (Maestría en Ciencias) - Departamento de Matemática Educativa Centro de Investigación y de Estudios Avanzados, Instituto Politécnico Nacional, Ciudad de México, 1989.

GIMÉNEZ, J., DIEZ- PALOMAR, J. Y CIVIL, M. Exclusión y Matemáticas. In: GIMÉNEZ J.; DÍEZPALOMAR J.; CIVIL M. (Comp.). Educación matemática y exclusión. Barcelona: Graó. 2007. p. 9-44.

GÓMEZ, K. Los procesos de difusión del conocimiento matemático en el cotidiano: un estudio socioepistemológico. 2009. 74f. Tesis (Maestría en Ciencias) - Departamento de Matemática Educativa Centro de Investigación y de Estudios Avanzados, Instituto Politécnico Nacional, Ciudad de México. 2009.

GÓMEZ, I. M. Procesos de aprendizaje en matemáticas con poblaciones de fracaso escolar en contextos de exclusión social: las influencias afectivas en el conocimiento de las matemáticas. 1997. 438 f. Tesis (Doctorado en Educación)- Facultad de Educación. Universidad Complutense de Madrid, Madrid, 1997.

GUERRERO, E.; BLANCO, L.; GIRL, N. El papel de la afectividad en la resolución de problemas matemáticos. Revista de Educación, Madrid, n. 340, p. 551-569, may./agos. 2006.

FERNANDES, S.H.A.A Y HEALY, L. Educação Matemática e Sociedade. Boletim de educação Matemática, Rio Claro, v. 23, n. 37, p. 1111 -1135, dic. 2010.

FERNÁNDEZ, J.M. La noción de violencia simbólica en la obra de Pierre Bourdieu: una aproximación crítica. Cuadernos de trabajo social, Madrid, n.18, p. 7-31. 2005.

KNIJNIK, G. Diversidad cultural, matemáticas y exclusión: oralidad y escritura en la educación matemática campesina del sur del Brasil. In: GIMÉNEZ J.; DÍEZ-PALOMAR J.; CIVIL M. (Comp.). Educación matemática y exclusión España: Graó, 2007. p. 63-82.

LARSON, R.; HOSTETLER, R.; EDWARDS, B. Cálculo y Geometría Analítica. México: Quinta Edición, McGraw-Hill. 1995.

L'HÔPITAL, A. Análisis de los infinitamente pequeños para el estudio de las líneas curvas. ed. Traducido por Cambray R. Ciudad de México: Servicios editoriales de la Facultad de Ciencias, UNAM. 1998. 
L'HOSPITAL, A. Analyse des infiniment petits pour l'intelligence de lignes courbe.

Paris: ACL-Editions. 1696

LITTLEWOOD, P. Escolarización exclusiva. In: LUENGO J. (Coord.). Paradigmas de gobernación y de exclusión social en la educación. Barcelona: Pomaires, 2005. p 67-90.

MONTIEL, G. Construcción de conocimiento trigonométrico: Un estudio Socioepistemológico. Ciudad de México: Ediciones Díaz de Santos. 2011.

PALACIOS, A.; HIDALGO, S.; MAROTO, A. Las aptitudes básicas como elemento determinante en el rendimiento en matemáticas: su influencia en los currículos de primaria. Revista de educación, Madrid, n. 320, p. 271-293, sep./dic.1999.

PERRENOUD, PH. La construcción del éxito y del fracaso escolar. Madrid: Morata. 1990.

POPKEWITZ, T.; LINDBLAD, S. Gobernación educativa e inclusión y exclusión social: dificultades conceptuales y problemáticas en la política y en la investigación. In: Luengo J. (Coord.). Paradigmas de gobernación y de exclusión social en la educación. Barcelona: Pomaires, 2005. p. 116-165.

PURCELL, E.; VARBERG, D. Cálculo con Geometría Analítica. México. Prentice Hall Hispanoamericana. 1995.

RIVAS, P. La Matemática como factor de deserción escolar y de exclusión social. Educere, Mérida,v. 9, n. 29, p. 175-170. 2005.

ROA-FUENTES. S Y OTACK, A. Validación de una descomposición genética de transformación lineal: un análisis refinado por la aplicación del ciclo de investigación de APOE. Revista latinoamericana de Investigación en Matemática Educativa, Ciudad de México, v. 15, n. 2.p. 199-232, jul. 2012.

SOTO, D. El Discurso Matemático Escolar y la Exclusión. Una Visión Socioepistemológica. 2010. Tesis (Maestría en Ciencias) - Departamento de Matemática Educativa Centro de Investigación y de Estudios Avanzados, Instituto Politécnico Nacional, Ciudad de México. 2010.

SOTO, D. Y REYES, D. En búsqueda de la exclusión en el discurso matemático escolar. Acta Latinoamericana de Matemática Educativa, México, v. 24, p. 873-880. 2011.

STEWART, J. Calculo: conceptos y contextos. Ciudad de México: Thomson. 1999.

TUYUB, I. y CANTORAL, R. Construcción social del conocimiento matemático durante la obtención de genes en una práctica toxicológica. Boletim de Educação Matemática, Rio Claro, v. 26, n. 42, p 311-328, abr. 2012.

ZALDÍVAR, J. Una caracterización de la función de un escenario de difusión de la ciencia desde una visión socioepistemológica: El caso de la resignificación de lo estable. 2009. 133f. Tesis (Maestría en Ciencias) - Departamento de Matemática Educativa Centro de Investigación y de Estudios Avanzados, Instituto Politécnico Nacional, Ciudad de México. 2009. 